\title{
Surgical management of tibial nerve schwannoma under (intraoperative neurophysiologic monitoring
}

\begin{abstract}
A schwannoma is a benign tumor originating from Schwann cells of the peripheral nerve sheath with a slow growth. The incidence of a schwannoma occurring in the foot and ankle is rare, with prevalence rate of $1 \%$ to $10 \%$. The removal of a schwannoma is easy but neurological complications can occur if care is not taken during dissection. In particular, a major neurological deficit such as motor weakness is critical in the lower limb and because of these risks the usage of IONM is unavoidable. In our case a 46years old male patient veteran of Iraq-Iran war presented with history of a slowly growing, swelling with dull aching pain over of the distal of right leg since 2 years was undergone neuromonitoring during our exploration surgery of tibia nerve.
\end{abstract}

Keywords: Intraoperative neurophysiology monitoring, schwannoma, tibia nerve
Volume 8 Issue 5 - 2018

\author{
Mehrabian Milad,' Dastan Sajed, ${ }^{2}$ Esmaeili \\ Melisa, ${ }^{3}$ Aghamolaei Darya, ${ }^{4}$ Babak Babapour ${ }^{5}$ \\ 'Department of Neurosurgery, Kerman University of Medical \\ Science, Iran \\ ${ }^{2}$ Department of Neurosurgery, Ludwing-Maximilians-University \\ (LMU), Germany \\ ${ }^{3}$ Department of Biomedical Engineer, Islamic Azad University \\ Central Tehran Branch, Iran \\ ${ }^{4}$ Department of Biomedical Engineer, Islamic Azad University \\ Central Tehran Branch, Iran \\ ${ }^{5}$ Neurosurgical Department, Medical School of Hannover, \\ Germany
}

Correspondence: Babak Babapour, Neurosurgical Department, Medical School of Hannover, Hannover, Germany, Email Info@Babapowr.Com

Received: January 21, 2018 | Published: October 26, 2018

\section{Introduction}

A schwannoma or neurilemmoma is a benign, and encapsulated tumor originating from Schwann cells of the peripheral nerve sheath. The incidence of a schwannoma occurring in the foot and ankle is rare, with prevalence rate of $1 \%$ to $10 \% .{ }^{1}$ Schwannomas are slow growing painless benign peripheral nerve sheath tumors that are rarely seen to occur in the lower limbs. Clinical presentation is often [misleading and ambiguous. ${ }^{2}$ The removal of a schwannoma can be easy in some cases; however, neurological complications can occur if care is not taken with the dissection. The threshold for iatrogenic injury during surgical dissection tends to be lower in the extremities than in the trunk. In particular, a major neurological deficit [such as motor weakness is critical in the lower limb. ${ }^{3}$ The value of routine use of intraoperative nerve monitoring (IONM) is clear for neurosurgeons in peripheral nerve lesions especially in lower extremities to reduce neurological deficits ${ }^{4}$ so it becomes .worldwide

\section{Patient and method}

A 46years old male patient veteran of Iraq-Iran war presented with history of a slowly growing, swelling with dull aching pain over of the distal of right leg since 2 years with paresthesia and numbness. Examination revealed a vertically oriented oval approximately $4 \times 4 \mathrm{~cm}$ in dimensions, solitary, firm, non-tender mass, which was not mobile in vertical direction and It was non-fluctuating. There was no sensory or motor deficit distal to the swelling. The patient's past medical and family history was no significant. Patient undergone exploration and excisional biopsy under general anesthesia previously to this admission. Under tourniquet control, a vertical skin incision was taken over the swelling and with subcutaneous dissection it was observed that the tumor was in close proximity \& along the tibial nerve. The tumor and the proximal and distal portions of the affected nerve were exposed (Figure A) (Figure B). Under loupe magnification, it was observed that the tumor was arising from the nerve sheath tissue and the nerve fibers crossed peripherally to the tumor. A longitudinal incision was carefully made in the epineurium.

The epineuria layers were gently peeled out until the yellow surface of the tumor was exposed. The entire tumor mass was subsequently shelled out under intraoperative nerve monitoring (IONM) without any damage to the fascicles \& was sent for histopathological laboratory. In this operation we used Nicolet vasys system and Ambu niddles to record ssep. Evoked intensity was 40ma. The patients' Post-operative recovery was with no neurological deficit under tight IONM Grossly, the surface of tumor was yellow (Figure B) and Microscopically (Figure $\mathrm{C}$ ), the tumor sections revealed a well circumscribed tumor comprised of hypercellular and hypocellular area (Antoni A and Antoni B).

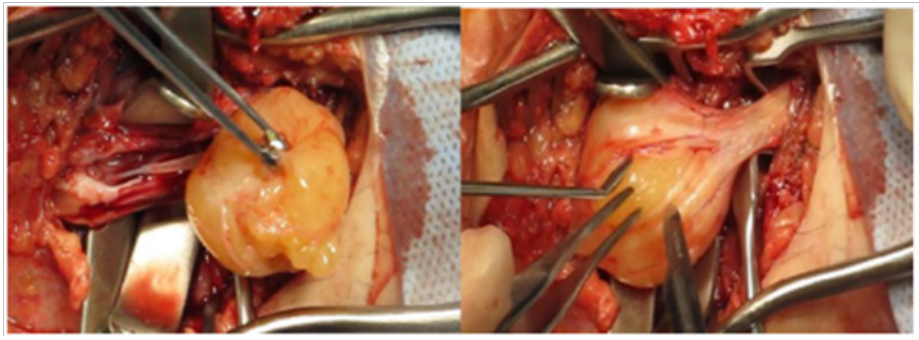

Figure A, B Proximal and distal portions of tumor. 


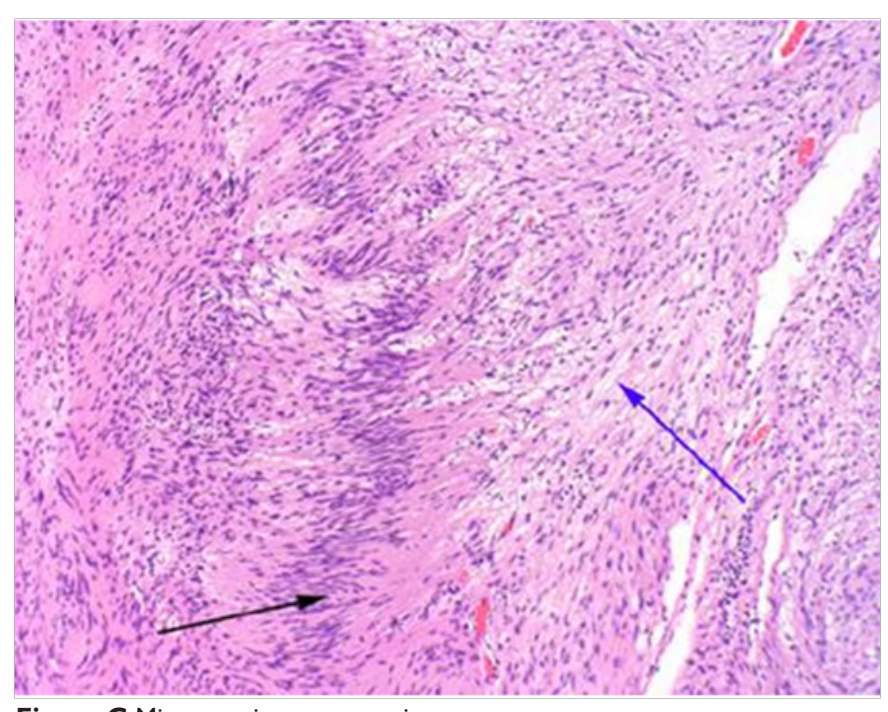

Figure C Microscopic tumor section.

\section{Discussion}

The tibia nerve is a mixed motor and sensory nerve, supplies motor function to the intrinsic foot muscles, as well as sensation of the medial heel and plantar foot. Tibial nerve dysfunction symptoms can include numbness, pain, tingling, and weakness of the knee or foot. slow growing benign peripheral nerve sheath tumors that are rarely seen in the lower limbs. ${ }^{2}$ In this case pain was the only symptom and it was important for surgeon to dissect the tumor without any neurological deficit, as Kim et al suggest that removal of schwannoma carried a risk of post-operative neurological deficit because of damage to fascicles. ${ }^{5}$ Use of IONM can abandon neurologic deficits during tumor resection.

\section{Conclusion}

Clinicians should consider schwannoma as a possible diagnosis for a well-defined, oval mass in patient with chronic pain and they should consider usage of IONM, even in dissection of benign tumors can reduce risk of neurological deficits.

\section{Acknowledgements}

None.

\section{Conflict of interest}

The author declares no conflict of interest.

\section{References}

1. Albert P, Patel J, Badawy K, et al. Peripheral Nerve Schwannoma: A Review of Varying Clinical Presentations and Imaging Findings. J Foot Ankle Surg. 2017;56(3):632-637.

2. Nawabi DH, Sinisi M. Schwannoma of the posterior tibial nerve: the problem of delay in diagnosis. J Bone Joint Surg Br. 2007;89:814-816.

3. Sang-Min Kim, Sung-Wook Seo. Surgical outcome of Schwannomas arising from major peripheral nerves in the lower limb. Int Orthop. 2012;36(8):1721-1725.

4. Anderson JC, Yamasaki DS. Intraoperative Nerve Monitoring During Nerve Decompression Surgery in the Lower Extremity. Clin Podiatric Med Surg. 2016;33(2):255-266.

5. Kim SM, See SW, Lee JY, et al. Surgical outcome of Schwannomas arising from major peripheral nerves in the lower limb. International Orthopaedics. 2012;36(8):1721-1725. 